\title{
A Method of Audio Digital Watermark Based on DWT
}

\author{
Yuanhang Cheng \\ College of Information Engineering \\ Shenyang University \\ Shenyang 110044, China \\ cyh518@163.com
}

\author{
Jing Wang \\ College of Information Engineering \\ Shenyang University \\ Shenyang 110044, China \\ sdwj91@163.com
}

\begin{abstract}
When a digital watermark is directly embedded into low frequency part of audio, sensitive noises maybe occur. In the paper, a method of audio digital watermark based on quantization index modulation is proposed to solve the problem. At first, audio signal is divided to frequency band subsections by audio mask effect. Then low frequency part of discrete wavelet transform (DWT) is extracted. Scramb-ling watermark is embedded into the low frequency part by quantization index modulation (QIM). The experimental results show that the presented method has a good capacity to fight many attacks.
\end{abstract}

Keywords-quantization index modulation; discrete wavelet transform; audio digital watermark

\section{INTRODUCTION}

In the information society, with the rapid development of multimedia and network technology, the transmitted information has the characteristics of openness and transparency in the internet. Sometimes, the transmitted information has privacy requirements, such as electronic signatures in electronic commerce, copyright certification of digital products, and protection of video and audio. Then, some new technologies appear, such as information hiding, digital fingerprint, Cryptography and digital Watermark[1][2]. By the technology of digital watermark, digital watermark can be embedded into multimedia without saving the authentication information. Digital watermark has better capacities of invisibility and anti-attack for the media better than other methods. With the wide popularity of music products, some requirements of copy restriction and piracy confirm for music products need be considered. In recent years, research on Audio Watermark is paid more attention[3][4].

In the technology of audio Watermark, the author information and background introduction associated with music production, as watermark, are embedded to audio signal IT CAN PROVIDE DIFFERENT basic platform for information retrieval based on content. Digital watermark based on DWT has good multi-resolution. By DWT, if the audio signal is divided to $\mathrm{L}$ layer, then $\mathrm{L}+1$ Band-pass filters of different bandwidth, which can be used to meet superposition, flexibility, time shift invariance synchronously, can be formed. Then the manner of embedding watermark can be selected flexibly, so it is widely applied in many digital watermark technologies. The technology based QIM has some advantages of anti-interference, good privacy, blind watermark detection, so it has also been widely applied.
In this paper, QIM [5] with DWT [6] are combined and applied to the audio digital watermark. The experiment result proves that the method proposed is effective.

\section{Discrete Wavelet Transform}

Usually, the high frequency components of DWT are directly embedded to watermark, WHICH can not be noticed by eyes/ears of the man. But high-frequency component is dealt with in the process of denoising and compression, The effect of extracted watermark is poor. Therefore, some experts consider that watermark is directly embedded to low frequency components of DWT [8,9]. Eye / ear are most sensitive on the low frequency components of images and audio. IF low frequency components of embedding digital watermark is attacked, which is easy to be noticed, and even can not by seen / heard, And thus lose the meaning of the digital watermark attacks but the method that watermark is directly embedded to low frequency components of DWT will produce aware sensitive noise of human eye/ears. So, watermark $\mathrm{w}(\mathrm{k})$ is embedded to signal $\mathrm{x}(\mathrm{k})$ according to the following guidelines.

The values of parameters $\alpha$ usually get $0 \leq \alpha \leq 1$. To improve the clarity of extracted watermark, parameters $\alpha$. will be increased. The sensitive noise will appear in the entire waveform because of low frequency components. If $\alpha$ is decreased, requirements of anti-interference can not be achieved. The method of DWT low-frequency components of the direct used,which is dilemma to improve the immunity and also to achieve the human ear can not hear the noise The quantization index modulation(QIM) form better effect of antiinterference through smaller quantization index of "pseudocoordinates". Therefore, DWT is combined with QIM, which can solve the problem of sensitive noise that watermark is directly embedded to low frequency components.

\section{DWT METHOD BASED QIM}

DWT based on QIM methods can be divided into: scrambling, dimension deduction of the watermark image, dividing fragment, DWT, quantization index modulation, inverse-DWT, interference imposed (such as compression, adding noise, low-pass, re-sampling), signal extraction, inverse -scrambling etc. Specific process is:

1) Scrambling: The data files of watermark can be scrambled by a transformation (such as Arnold Transformation 
etc) in order to ensure the invisibility of watermark information Taking into the relatively long running time of DWT, to improve processing power, this article uses Fibonacci Transform [10] of simple transformation (only have $n(n-1)$ kind, $\mathrm{n}$ is order )and quickly scrambling to improve processing capacity .

2) Reducing the dimensions: Because embedding watermark adopt two-dimensional image, watermark image $\mathrm{P}(\mathrm{M}, \mathrm{N})$ can be showed as:

$$
p(i, j), 1 \leq i \leq M, 1 \leq j \leq N
$$

The watermark will be embedded to one-dimensional audio signal $\mathrm{S}(\mathrm{k})$, its dimensions must be reduced, that is

$$
w=\{w(k)=p(i, j), 1 \leq i \leq M, 1 \leq j \leq N, k=i * N+j\}
$$

Then, watermark is binary and one-dimensional scrambling watermark sequence is formed.

3) Dividing fragment by Masking effect: Because ears is the most sensitive to audio signal sequence $\mathrm{x}(\mathrm{k})$ between $300 \sim 3$ $400 \mathrm{~Hz}$., low frequency components following in the $2 \mathrm{kHz}$ is very plentiful, distortion generated by the subsequent band can be masked out, the band above $4 \mathrm{kHz}$ can be easily destroyed by various signal processing methods, and affect the robustness of the watermark. Therefore, the watermark signal is embedded into audio signal between $2 \mathrm{kHz}$ to $4 \mathrm{kHz}$ band, let it as $\mathrm{x}^{\prime}(\mathrm{k})$. Audio data sequence $\mathrm{x}^{\prime}(\mathrm{k})$ is divided into fragment $\mathrm{L}$ according to data length. If audio data is not integer multiples of L, audio data make up a small 0 to meet the requirements of pseudocoordinates formed by QIM

4) Determine the low-frequency component of DWT: Audio signals $x^{\prime}(k)$ that have been divided into fragment take low-frequency component $\mathrm{s}(\mathrm{K})$ of DWT. The low-frequency component is normalized for QIM
5) Quantization index modulation (QIM). Random signal $\mathrm{r} 1(\mathrm{~K})$ is produced at first,

$$
K=1,2, \ldots, L^{*} k, 0 \leq r 1(K) \leq 1
$$

In order to make quantify the indexer $\mathrm{r} 1$ and $\mathrm{r} 2$ correspond 0 and 1 of watermark signal $\mathrm{w}(\mathrm{k})$, take $\mathrm{rl}(\mathrm{K})[7]$ by the following rules

$$
\left\{\begin{array}{l}
r_{2}(K)=r_{1}(K)+\lambda / 2, K=1,2, \cdots, L^{*} K, \text { ifr }_{1}(K)<0 \\
r_{2}(K)=r_{1}(K)-\lambda / 2, K=1,2, \cdots, L^{*} K, \text { ifr }_{2}(K) \geq 0
\end{array}\right.
$$

Which $\lambda=0.5$ is best, experiments show if $\lambda>0.5$ or $\lambda<0.5$ can not guarantee to meet $0 \leq \mathrm{r} 2(\mathrm{~K}) \leq 1$ for all $\mathrm{K}$.

Method of embedding watermark is: If $\mathrm{q}$ is linear quantizer, $\mathrm{q} 1$ and q2 [7] is defined as follows

$$
\left\{\begin{array}{l}
q_{1}(K)=q\left[x(K)+r_{1}\right]-r_{1}, K=1,2, \cdots, L^{*} K, \text { if } w(K)=0 \\
q_{2}(K)=q\left[x(K)+r_{2}\right]-r_{2}, K=1,2, \cdots, L^{*} K, i f w(K)=1
\end{array}\right.
$$

Therefore, $\mathrm{x}(\mathrm{k})$ is replaced with $\mathrm{q} 1$ and $\mathrm{q} 2$ to complete the process of embedding watermark to the low frequency components .

\section{EXPERIMENTAL RESULTS AND ANALYSIS}

The experiments of quantization index modulation is carried out to voice files of 9 seconds (Fs $=11025,88 \mathrm{bps}$ ) and popular music files of 7 seconds ( $F=22050,176 \mathrm{bps})$. The embedding watermark image is $64 \times 64$ standard image after binaryzation (Shenyang University .bmp). The extracted watermark is clearer after exerting Gaussian noise of SNR $=30$ to voice files. The experimental result is shown as Fig.1.
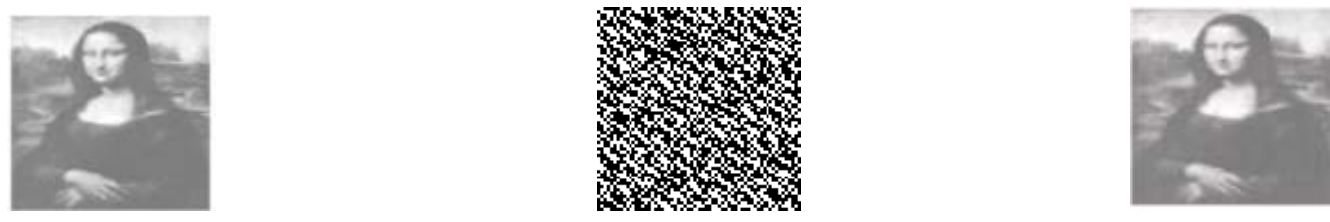

scrambling watermark

recovered watermark
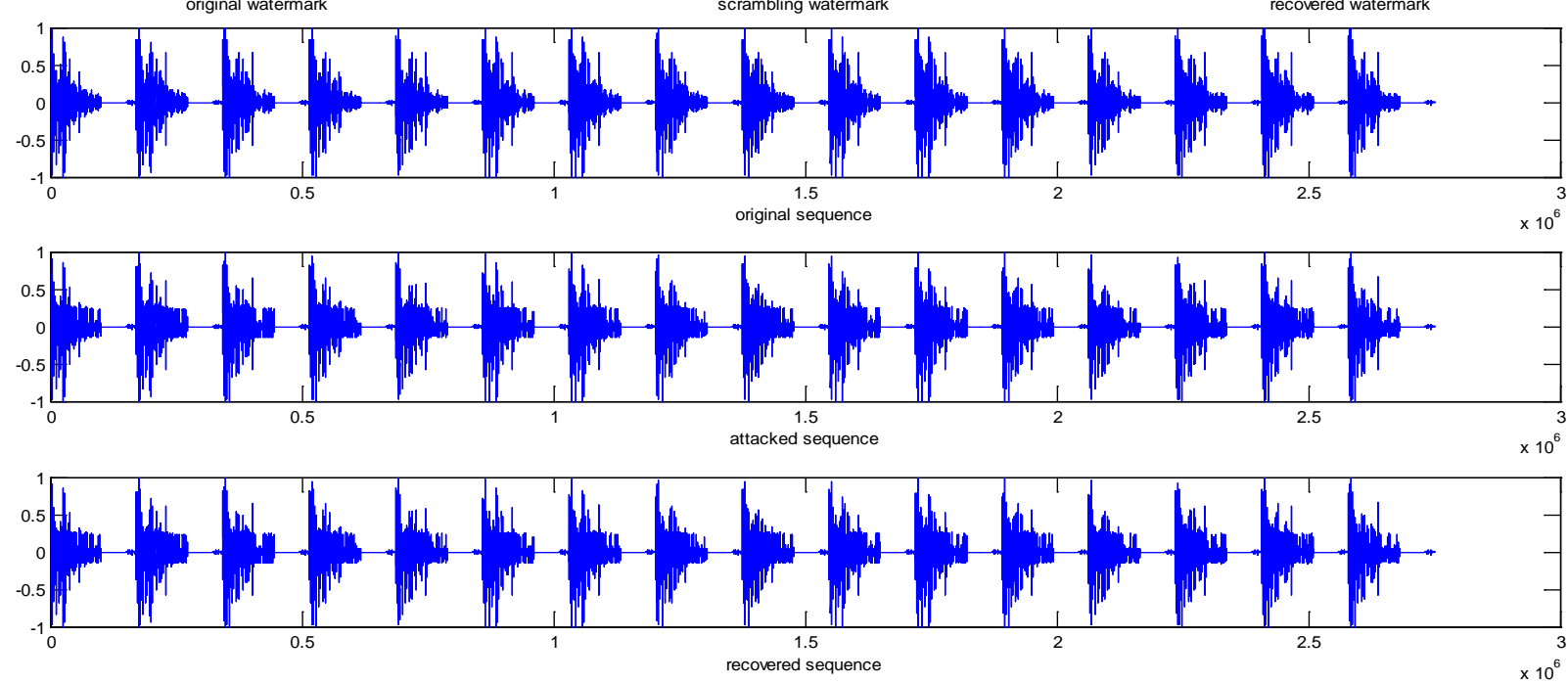

Fig.1. The result of voice files (The number of iterations scrambling $=7$ ) 
The extracted watermark is clearer after exerting continuously resampling, cutting, Gaussian noise, lossy

compression to popular music files. The result is shown as Fig.2.
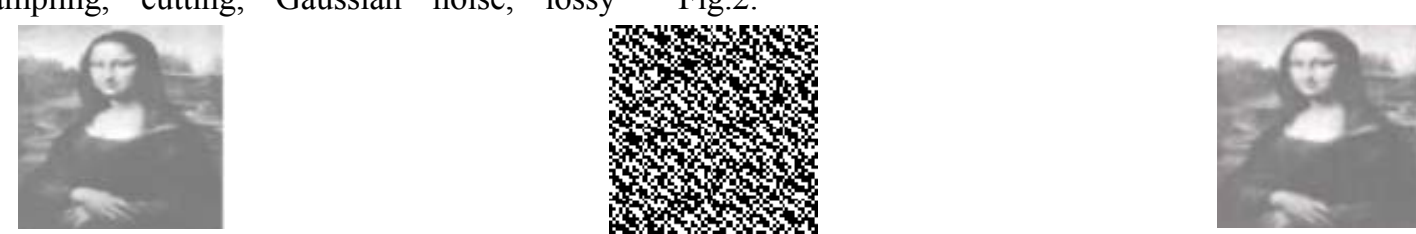

original watermark

scrambling watermark

recovered watermark
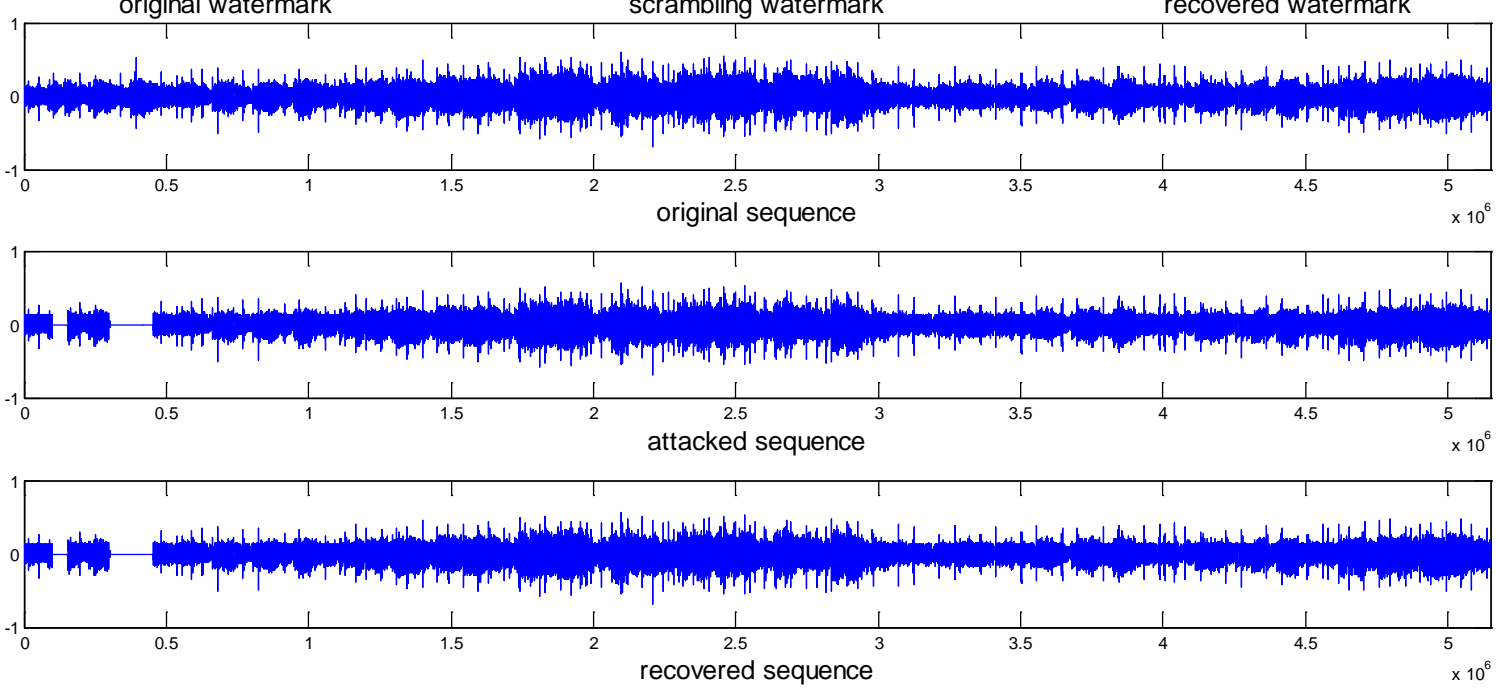

Fig.2. The result of popular music files (The number of iterations scrambling=11)

Fig.1 and Fig.2 were showed that signal of embedding watermark is the same compared with original signal in the different audio files, the human ears can not distinguish the difference.

\section{CONCLUSION}

The experiment shows that the method of audio digital watermark based on QIM can extract watermark image correctly and solve the problem of sensitive noise. It is caused of watermark embedded into low frequency part directly, carrying out different attacks and exerting a strong variety of offensive interference to audio signal. The experiment shows that this method has better robustness to many attacks.

\section{REFERENCES}

[1] W Bender D, Gruhl N, Morimoto A Lu, "Techniques for data hiding," IBM SYSTEMS JOURNAL,vol.35,pp.3-4,1996.

[2] Mei S. chun; Li R. hou; Dang H. mei, "Digital image watermarking algorithm based on phase shift keying," J.Ivffcro Systems, vol.26,pp.485-488,2005.

[3] Huang Jiwu, Shi Yanqing, "Embedding image watermarking in DC components," Transactions on Circuits and Systems for Video Technology, vol.10, pp.974-979,2000.

[4] LI Xu dong, "Mean gray value based image watermarking algorithm," Geomatics and Information Science of Wuhan University, vol.32, pp. 556-559,2007.

[5] Chen, B\&Wornell, G.. W. B. Quantization index modulation, “ A class of provably good methods for digital watermarking and information embedding,"IEEE Trans Information Theory,vol.47,pp.1423-1443, 2001

[6] Wang Ying, Xiao Jun, Wang Yun-hong, "Principles and techniques of digital watermarking,"M. Bei Jing: Science Press, 2007. 